Supplement of Biogeosciences, 12, 2131-2151, 2015

http://www.biogeosciences.net/12/2131/2015/

doi:10.5194/bg-12-2131-2015-supplement

(C) Author(s) 2015. CC Attribution 3.0 License.

(c) (i)

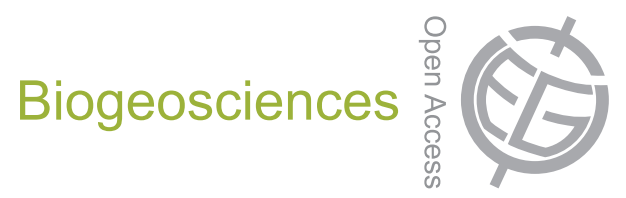

Supplement of

\title{
Reconstruction of secular variation in seawater sulfate concentrations
}

\section{T. J. Algeo et al.}

Correspondence to: T. J. Algeo (thomas.algeo@uc.edu) 
Table 1. MSR fractionation data for modern aqueous systems

\begin{tabular}{|c|c|c|c|c|c|c|c|c|}
\hline Rec. & Site & $\begin{array}{c}\text { Env } \\
\text { Type }^{a}\end{array}$ & $\begin{array}{c}\delta^{34} S- \\
\text { sulfate }\end{array}$ & $\begin{array}{c}\delta^{34} S- \\
\text { sulfide }^{b}\end{array}$ & $\begin{array}{c}\Delta^{34} S_{\text {sulf- }} \\
\text { py }\end{array}$ & $\begin{array}{l} \pm 1 \\
\text { s.d. }\end{array}$ & {$\left[\mathrm{SO}_{4}{ }^{2-}\right]$} & Reference \\
\hline & & & $(\%)$ & $(\%)$ & $(\%)$ & $(\%)$ & $(\mu \mathrm{M})$ & \\
\hline 1 & Linsley Pond, Conn. & FW-O & 7.7 & $-1.0+$ & 8.7 & & 45 & Nakai \& Jensen, 1964 \\
\hline 2 & Queechy Pond, Conn. & FW-O & 5.8 & $4.6+$ & 1.2 & & 48 & Nakai \& Jensen, 1964 \\
\hline 3 & Mt. Tom Pond, Conn. & FW-O & 6.1 & $-3.0+$ & 9.1 & & 53 & Nakai \& Jensen, 1964 \\
\hline 4 & Lake Fukami-ike, Japan & FW-O & -15.0 & $-22.0 \ddagger$ & 7.0 & & $4(17$ & Nakagawa et al., 2012 \\
\hline 5 & Lake Ontario & FW-O & 6.9 & $-1.2+$ & 8.1 & & 302 & Nriagu \& Coker, 1976 \\
\hline 6 & McFarlane Lake, Ontario & FW-O & 6.0 & -3.0 & 9.0 & & 250 & Nriagu \& Soon, 1985; Nriagu \& Harvey, 1978 \\
\hline 7 & Kelley Lake, Ontario & FW-O & 5.0 & -10.0 & 15.0 & & 5000 & Nriagu \& Soon 1985 \\
\hline 8 & Turkey Lake, Ontario & FW-O & 6.0 & 2.0 & 4.0 & & 55 & Nriagu \& Soon 1985 \\
\hline 9 & Batchawana Lake, Ontario & FW-O & 5.0 & 2.0 & 3.0 & & 55 & Nriagu \& Soon 1985 \\
\hline 10 & Okefenokee Swamp, Georgia & FW-O & 9.0 & 6.2 & 2.8 & 4.0 & 521 & Price \& Casagrande, 1991 \\
\hline 11 & New Jersey Pinelands-swamp & FW-O & 5.0 & $-8.8^{*}$ & 13.8 & 5.0 & 400 & Mandernack et al., 2000 \\
\hline 12 & Lake Biwa, Japan & FW-O & 2.3 & $-4.0 \ddagger$ & 6.3 & 1.5 & 110 & Karube et al., 2012; Nakano et al., 2008 \\
\hline 13 & Everglades, Florida (2 cores) & FW-O & 17.0 & 10.2 & 6.8 & & 530 & Bates et al., 1998 \\
\hline 14 & Mud Lake, Florida (2cores) & FW-O & 12.0 & 4.3 & 7.7 & & 1000 & Bates et al., 1995 \\
\hline 15 & Hufeisensee, Germany & FW-E & 1.8 & $-4.1^{*}$ & 5.9 & & 1200 & Asmussen \& Strauch, 1998 \\
\hline 16 & Lago di Cadagno, Switzerland & FW-E & 27.0 & -8.0 & 35.0 & & 2000 & Canfield et al., 2010 \\
\hline 17 & Steisslingensee, Germany & FW-E & -6.0 & -15.0 & 9.0 & & 490 & Mayer \& Schwark, 1999 \\
\hline 18 & McCarrons Lake, MN & FW-E & 5.0 & -0.1 & 5.1 & & 302 & Gomes \& Hurtgen, 2013 \\
\hline 19 & Aarhus Bay, Denmark & BW-O & 21.6 & -35.5 & 56.5 & 4.5 & 20747 & Johnston et al., 2008 \\
\hline 20 & Apalachicola Bay, Florida (EB) & BW-O & 12.0 & $-24.0 \ddagger$ & 36.0 & 7.0 & 4149 & Chanton \& Lewis, 1999 \\
\hline 21 & Apalachicola Bay, Florida (CP-DB) & BW-O & 18.0 & $-13.5 \ddagger$ & 31.5 & 7.0 & 16598 & Chanton \& Lewis, 1999 \\
\hline 22 & Monie Bay, Maryland (HWY) & BW-O & 22.5 & $2.4 \ddagger$ & 20.1 & & 300 & Stribling et al., 1998 \\
\hline 23 & Monie Bay, Maryland (DB2) & BW-O & 21.7 & $-11.1 \ddagger$ & 32.8 & & 6000 & Stribling et al., 1998 \\
\hline 24 & Monie Bay (BAY) & $\mathrm{BW}-\mathrm{O}$ & 20.8 & $-22.4 \ddagger$ & 43.2 & & 12448 & Stribling et al., 1998 \\
\hline 25 & Monie Bay (DB1) & $\mathrm{BW}-\mathrm{O}$ & 21.7 & $-2.5 \ddagger$ & 24.2 & & 6000 & Stribling et al., 1998 \\
\hline 26 & Jade Bay, Waddensee, Germany & $\mathrm{BW}-\mathrm{O}$ & 20.5 & -22.5 & 43.0 & & 22500 & Llobet-Brossa et al., 2002 \\
\hline 27 & Baltic Sea & $\mathrm{BW}-\mathrm{O}$ & & & 23.5 & 9.5 & 8299 & Lein, 1983 \\
\hline 28 & Black Sea & BW-O & 21.5 & -46.8 & 68.0 & 3.0 & 16000 & Johnston et al., 2008 \\
\hline 29 & Black Sea & BW-O & 18.5 & -33.0 & 51.5 & 12.0 & 16000 & Wijsman et al., 2001 \\
\hline
\end{tabular}




\begin{tabular}{|c|c|c|c|c|c|c|c|c|}
\hline 30 & Logten Lagoon, Denmark & $\mathrm{BW}-\mathrm{O}$ & 26.0 & -7.0 & 33.0 & & 13000 & Habicht \& Canfield, 1997 \\
\hline 31 & Everglades, Florida-Little Shark River & BW-O & 17.0 & -28.0 & 45.0 & 5.0 & 21875 & Price \& Casagrande, 1991 \\
\hline 32 & Everglades, Florida-Mud Bay & $\mathrm{BW}-\mathrm{O}$ & 12.0 & 3.0 & 9.0 & 2.0 & 3125 & Price \& Casagrande, 1991 \\
\hline 33 & Sapelo Island, GA & $\mathrm{BW}-\mathrm{O}$ & 21.0 & $-19.0 *$ & 40.0 & & 19500 & Peterson \& Howarth, 1987 \\
\hline 34 & Lake Chany, Russia & $\mathrm{BW}-\mathrm{O}$ & 29.8 & $16.6 \ddagger$ & 18.6 & & 2240 & Doi et al., 2004 \\
\hline 35 & Long Island Sound, Conn. & $\mathrm{BW}-\mathrm{O}$ & 20.5 & $-12.5^{\dagger}$ & 33.0 & 0.5 & 7542 & Nakai \& Jensen, 1964 \\
\hline 36 & Branford Bay, Conn. & $\mathrm{BW}-\mathrm{O}$ & 20.4 & $-21.3+$ & 41.7 & 0.5 & 6802 & Nakai \& Jensen, 1964 \\
\hline 37 & Green Lake, NY & BW-E & 24.6 & $-17.7 *$ & 55.8 & & 13542 & Nakai \& Jensen, 1964; Suits \& Wilkin, 1998 \\
\hline 38 & Fayetteville Green Lake, NY & BW-E & 25.9 & $-30.9 *$ & 56.8 & & 15000 & Fry, 1986a, 1986b; Fry et al., 1995 \\
\hline 39 & Fayetteville Green Lake, NY & BW-E & 32.0 & $-24.0 *$ & 57.0 & & 15000 & Zerkle et al., 2010 \\
\hline 40 & Lake Mogil'noe, Russia & BW-E & 30.0 & $-27.0 *$ & 57.0 & & 21875 & Ivanov et al., 2001 \\
\hline 41 & Lake Mogil'noe, Russia & BW-E & & $*$ & 39.5 & 7.5 & 21875 & Gorlenko et al., 1978 \\
\hline 42 & Baltic Sea & BW-E & & & 35.0 & 11.0 & 9959 & Lein, 1983 \\
\hline 43 & Black Sea & BW-E & 19.5 & -38.2 & 57.7 & 2.5 & 17500 & Wijsman et al., 2001 \\
\hline 44 & Black Sea & BW-E & & & 50.5 & 7.5 & 17500 & Lyons, 1997; Wijsman et al., 2001 \\
\hline 45 & Black Sea & BW-E & & $*$ & 59.0 & 2.0 & 17500 & Sweeney \& Kaplan, 1980; Wijsman et al., 2001 \\
\hline 46 & Black Sea & BW-E & 23.9 & -38.0 & 61.9 & 4.0 & 17500 & Johnston et al., 2008; Wijsman et al., 2001 \\
\hline 47 & Black Sea & BW-E & & $*$ & 62.0 & & 17500 & Fry et al., 1991; Wijsman et al., 2001 \\
\hline 48 & Framvaren Fjord & BW-E & 21.0 & $-22.8^{*}$ & 43.8 & 2.3 & 18550 & Mandernack et al., 2003 \\
\hline 49 & Gotland Deep, Baltic Sea & BW-E & 20.0 & -26.0 & 46.0 & 6.0 & 9959 & Sternbeck \& Sohlenius, 1997 \\
\hline 50 & Kiel Bay, Baltic Sea & BW-E & 20.0 & $-29.4 \ddagger$ & 49.4 & 2.0 & 19565 & Hartmann \& Nielsen, 1968 \\
\hline 51 & Maringer Fjord, Norway & BW-E & 20.5 & $-16.0 *$ & 36.5 & 6.0 & 13000 & Sørensen \& Canfield, 2004 \\
\hline 52 & Lake Sakovo, Russia & BW-E & 14.0 & -11.0 & 27.0 & & 8125 & Matrosov et al., 1975; Gorlenko \& Chebotarev, 1981 \\
\hline 53 & Chernyi Kichiyer (Black Kichier) & BW-E & & & 23.0 & & 896 & Matrosov et al., 1975 \\
\hline 54 & Bol'shoy Kichiyer (Big Kichier) & BW-E & & & 4.1 & & 448 & Matrosov et al., 1975 \\
\hline 55 & FOAM, Long Island Sound, NY & SW-O & & -29.0 & 49.0 & & 23237 & $\begin{array}{l}\text { Canfield et al., 1992; Canfield \& Thamdrup, 1994; } \\
\text { Lee \& Lwiza, } 2005\end{array}$ \\
\hline 56 & Black Hole, Long Island Sound & SW-O & & & 41.0 & & 20747 & Canfield \& Thamdrup, 1994; Lee \& Lwiza, 2005 \\
\hline 57 & NW Control, Long Island Sound, NY & SW-O & & & 58.0 & & 23237 & Canfield \& Thamdrup, 1994; Lee \& Lwiza, 2005 \\
\hline 58 & Sachem, Long Island Sound, NY & SW-O & & & 34.0 & & 23237 & Canfield \& Thamdrup, 1994; Lee \& Lwiza, 2005 \\
\hline 59 & Pearl River Delta, China & SW-O & 20.5 & $-20.0 \ddagger$ & 40.5 & & 27386 & Böttcher et al., 2010 \\
\hline 60 & San Diego Trough, California Shelf & SW-O & 20.4 & -28.8 & 49.2 & & 27500 & Kaplan et al., 1963 \\
\hline 61 & Newport Marsh, California & SW-O & 20.4 & -20.0 & 40.4 & & 23571 & Kaplan et al., 1963 \\
\hline 62 & St Andrew Bay, Florida & SW-O & 21.5 & -25.8 & 47.3 & 4.0 & 24897 & Brüchert and Pratt, 1996 \\
\hline 63 & St Andrew Bay, Florida (WB) & SW-O & 21.5 & -15.5 & 37.0 & & 28000 & Brüchert and Pratt, 1999 \\
\hline
\end{tabular}




\begin{tabular}{|l|l|r|r|r|r|r|r|l|}
\hline 64 & St Andrew Bay, Florida (CB) & SW-O & 21.5 & -19.2 & 40.7 & & 22800 & Brüchert and Pratt, 1999 \\
\hline & & & & & & & & \\
\hline 76 & Makirina Bay, Croatia & HY-O & 21.0 & -29.0 & 50.0 & 2.0 & 34025 & Lojen et al., 2004 \\
\hline 77 & Solar Lake, Egypt & HY-O & 23.4 & -20.8 & 44.2 & 2.0 & 126000 & Johnston et al., $2008 ;$ Jorgensen \& Cohen, 1977 \\
\hline 78 & Solar Lake, Egypt & HY-O & 22.0 & -17.0 & 39.0 & & 65000 & Habicht \& Canfield, $1997 ;$ Jorgensen \& Cohen, 1977 \\
\hline 79 & Lake Vanda, Antarctica (deep) & HY-E & 46.0 & $13.9^{*}$ & 32.1 & & 55187 & Purdy et al., 2001 \\
\hline 80 & Lake Vanda, Antarctica (deep) & HY-E & 46.0 & $10.5^{*}$ & 35.5 & & 55187 & Nakai et al., 1975 \\
\hline 81 & Mahooney Lake, B.C., Canada & HY-E & 27.5 & $-24.1^{*}$ & 51.6 & 0.5 & 420000 & Overmann et al., 1996 \\
\hline
\end{tabular}

Notes:

${ }^{a}$ Environment types are hypersaline (HY)( $\left.>40 \mathrm{psu}\right)$; seawater (SW)(30-40 psu); brackish water (BW)(10-30 psu); and freshwater (FW)( $\left.<10 \mathrm{psu}\right)$. Depositional environments are further classified as containing oxic $(\mathrm{O})$ or euxinic $(\mathrm{E})$ porewaters.

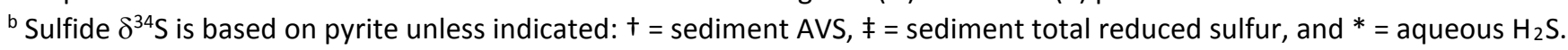


Table 2. Phanerozoic $\delta^{34} S_{\text {CAS }}$ data (used to generate Table 3)

\begin{tabular}{|c|c|c|c|}
\hline \multicolumn{2}{|c|}{ Paytan et al. (1998) } & \multicolumn{2}{|c|}{ Kampschulte \& Strauss (2004) } \\
\hline Age & $\delta^{34} S_{\text {CAS }}$ & Age & $\delta^{34} S_{\text {CAS }}$ \\
\hline (Ma) & $(\%)$ & (Ma) & (\%o) \\
\hline 00 & 209 & 1300 & 153 \\
\hline 0.0 & 21.0 & 132.0 & 15.9 \\
\hline 0.0 & 21.1 & 133.0 & 17.0 \\
\hline 0.0 & 21.1 & 134.0 & 18.0 \\
\hline 0.0 & 21.3 & 134.0 & 17.4 \\
\hline 0.0 & 21.4 & 134.0 & 16.2 \\
\hline 0.2 & 20.9 & 136.0 & 18.5 \\
\hline 0.2 & 21.1 & 136.0 & 15.2 \\
\hline 2.2 & 22.0 & 136.0 & 16.7 \\
\hline 2.6 & 22.0 & 138.0 & 16.5 \\
\hline 3.8 & 21.9 & 139.0 & 16.1 \\
\hline 4.8 & 22.3 & 140.0 & 25.0 \\
\hline 5.2 & 21.8 & 140.0 & 21.8 \\
\hline 5.7 & 22.1 & 140.0 & 14.8 \\
\hline 6.0 & 22.0 & 142.0 & 13.3 \\
\hline 6.4 & 22.3 & 144.0 & 12.7 \\
\hline 6.8 & 22.3 & 154.0 & 16.1 \\
\hline 7.8 & 21.8 & 157.0 & 15.3 \\
\hline 7.8 & 22.0 & 161.0 & 16.6 \\
\hline 8.1 & 22.3 & 161.0 & 15.8 \\
\hline 9.3 & 21.8 & 164.0 & 17.5 \\
\hline 9.7 & 22.1 & 167.0 & 20.7 \\
\hline 10.2 & 21.9 & 170.0 & 18.5 \\
\hline 11.4 & 22.2 & 173.0 & 18.1 \\
\hline 12.1 & 22.1 & 178.0 & 18.0 \\
\hline 12.6 & 21.9 & 184.0 & 23.6 \\
\hline 12.6 & 22.0 & 187.0 & 17.4 \\
\hline 12.7 & 22.0 & 197.0 & 14.3 \\
\hline 12.7 & 22.7 & 208.0 & 24.4 \\
\hline 12.8 & 22.2 & 210.0 & 18.5 \\
\hline 12.9 & 22.7 & 211.0 & 18.0 \\
\hline 13.4 & 22.1 & 214.0 & 19.0 \\
\hline 13.7 & 22.0 & 216.0 & 17.4 \\
\hline 14.2 & 21.7 & 221.0 & 19.2 \\
\hline 15.1 & 22.0 & 225.0 & 18.5 \\
\hline 15.1 & 22.1 & 237.0 & 17.5 \\
\hline 15.7 & 22.4 & 240.0 & 20.1 \\
\hline 16.3 & 21.8 & 242.0 & 26.4 \\
\hline 16.3 & 22.0 & 245.0 & 16.7 \\
\hline
\end{tabular}




\begin{tabular}{|c|c|c|c|}
\hline 17.2 & 22.1 & 245.0 & 15.7 \\
\hline 18.1 & 21.9 & 246.0 & 24.5 \\
\hline 19.0 & 21.8 & 253.0 & 10.9 \\
\hline 20.0 & 21.6 & 264.0 & 12.5 \\
\hline 21.0 & 22.0 & 289.0 & 12.5 \\
\hline 22.2 & 22.0 & 295.0 & 11.8 \\
\hline 23.5 & 21.9 & 297.0 & 12.3 \\
\hline 24.1 & 21.9 & 298.0 & 11.0 \\
\hline 25.6 & 21.7 & 304.0 & 12.9 \\
\hline 26.4 & 21.4 & 305.0 & 13.3 \\
\hline 26.4 & 21.8 & 306.0 & 13.8 \\
\hline 26.4 & 21.9 & 306.0 & 12.6 \\
\hline 27.4 & 21.4 & 309.0 & 12.6 \\
\hline 28.5 & 21.2 & 310.0 & 13.0 \\
\hline 29.0 & 21.3 & 311.0 & 15.4 \\
\hline 29.0 & 21.5 & 313.0 & 15.1 \\
\hline 29.5 & 21.7 & 316.0 & 15.4 \\
\hline 30.0 & 21.6 & 316.0 & 14.5 \\
\hline 30.8 & 21.4 & 317.0 & 15.0 \\
\hline 31.0 & 21.7 & 318.0 & 16.8 \\
\hline 32.6 & 21.6 & 318.0 & 15.0 \\
\hline 33.7 & 22.0 & 319.0 & 15.7 \\
\hline 33.8 & 21.8 & 321.0 & 17.5 \\
\hline 34.2 & 21.6 & 321.0 & 16.7 \\
\hline 34.6 & 21.8 & 324.0 & 15.7 \\
\hline 35.1 & 22.4 & 324.0 & 14.7 \\
\hline 35.1 & 22.5 & 324.0 & 13.5 \\
\hline 35.6 & 22.2 & 326.0 & 16.2 \\
\hline 36.5 & 22.5 & 326.0 & 15.2 \\
\hline 37.5 & 22.1 & 327.0 & 12.1 \\
\hline 37.5 & 22.3 & 329.0 & 14.1 \\
\hline 39.4 & 22.2 & 330.0 & 15.3 \\
\hline 40.5 & 22.3 & 331.0 & 14.0 \\
\hline 41.7 & 22.1 & 331.0 & 13.8 \\
\hline 43.8 & 22.4 & 331.0 & 12.8 \\
\hline 44.5 & 21.9 & 332.0 & 15.0 \\
\hline 44.5 & 22.0 & 334.0 & 14.5 \\
\hline 46.0 & 21.6 & 334.0 & 13.7 \\
\hline 46.0 & 21.5 & 335.0 & 12.9 \\
\hline 48.2 & 20.3 & 336.0 & 15.6 \\
\hline 49.7 & 19.1 & 337.0 & 14.5 \\
\hline 49.9 & 19.3 & 338.0 & 12.8 \\
\hline 50.2 & 19.1 & 339.0 & 13.4 \\
\hline 51.2 & 18.7 & 343.0 & 14.6 \\
\hline 51.6 & 18.0 & 343.0 & 13.7 \\
\hline 51.9 & 18.1 & 345.0 & 15.9 \\
\hline
\end{tabular}




\begin{tabular}{|c|c|c|c|}
\hline 53.6 & 17.8 & 346.0 & 21.2 \\
\hline 55.5 & 17.4 & 349.0 & 17.7 \\
\hline 56.4 & 17.7 & 351.0 & 18.5 \\
\hline 57.0 & 17.5 & 353.0 & 17.6 \\
\hline 57.6 & 18.2 & 355.0 & 23.3 \\
\hline 57.6 & 18.3 & 355.0 & 21.3 \\
\hline 57.9 & 17.9 & 360.0 & 20.6 \\
\hline 57.9 & 18.0 & 360.0 & 19.7 \\
\hline 59.2 & 18.1 & 380.0 & 22.7 \\
\hline 60.7 & 18.6 & 380.0 & 22.0 \\
\hline 60.9 & 19.0 & 383.0 & 16.4 \\
\hline 61.8 & 19.1 & 386.0 & 17.3 \\
\hline 63.9 & 18.8 & 391.0 & 23.3 \\
\hline 65.2 & 19.0 & 403.0 & 24.5 \\
\hline 65.2 & 18.9 & 406.0 & 28.5 \\
\hline 65.5 & 19.1 & 408.0 & 24.4 \\
\hline 66.0 & 18.8 & 413.0 & 26.6 \\
\hline 66.8 & 18.8 & 422.0 & 25.8 \\
\hline 68.7 & 18.9 & 426.0 & 25.8 \\
\hline 68.7 & 18.9 & 426.0 & 24.8 \\
\hline 70.0 & 18.8 & 426.0 & 24.5 \\
\hline 71.3 & 19.1 & 427.0 & 27.5 \\
\hline 73.0 & 19.2 & 427.0 & 23.4 \\
\hline 74.2 & 19.1 & 432.0 & 24.3 \\
\hline 74.4 & 19.3 & 434.0 & 30.2 \\
\hline 75.3 & 19.4 & 435.0 & 28.9 \\
\hline 75.6 & 19.3 & 436.0 & 35.6 \\
\hline 76.4 & 19.1 & 436.0 & 29.2 \\
\hline 78.4 & 19.0 & 436.0 & 28.2 \\
\hline 78.8 & 19.1 & 436.0 & 27.6 \\
\hline 80.3 & 19.0 & 437.0 & 31.5 \\
\hline 82.0 & 18.9 & 438.0 & 26.2 \\
\hline 83.0 & 18.2 & 439.0 & 14.5 \\
\hline 83.6 & 18.4 & 441.0 & 24.3 \\
\hline 83.7 & 18.4 & 441.0 & 24.0 \\
\hline 83.9 & 18.1 & 443.0 & 27.0 \\
\hline 85.6 & 18.3 & 443.0 & 22.6 \\
\hline 88.4 & 18.3 & 445.0 & 31.6 \\
\hline 88.4 & 18.1 & 445.0 & 21.8 \\
\hline 91.0 & 18.6 & 445.0 & 21.1 \\
\hline 93.0 & 18.9 & 446.0 & 23.0 \\
\hline 93.4 & 19.1 & 447.0 & 32.9 \\
\hline 93.5 & 19.0 & 454.0 & 29.3 \\
\hline 93.6 & 18.8 & 454.0 & 24.5 \\
\hline 93.8 & 19.0 & 456.0 & 27.4 \\
\hline 95.0 & 19.2 & 461.0 & 22.9 \\
\hline
\end{tabular}




\begin{tabular}{|c|c|c|c|c|}
\hline 95.8 & 19.0 & & 468.0 & 19.1 \\
\hline 97.0 & 19.1 & & 471.0 & 26.8 \\
\hline 97.0 & 18.5 & & 472.0 & 28.7 \\
\hline 98.9 & 17.9 & & 475.0 & 27.8 \\
\hline 98.9 & 17.8 & & 475.0 & 25.8 \\
\hline 100.0 & 16.3 & & 477.0 & 17.6 \\
\hline 104.0 & 15.6 & & 478.0 & 26.8 \\
\hline 104.0 & 15.6 & & 484.0 & 29.0 \\
\hline 107.0 & 15.7 & & 484.0 & 27.6 \\
\hline 108.0 & 15.9 & & 486.0 & 32.3 \\
\hline 109.0 & 16.1 & & 491.0 & 30.5 \\
\hline 110.0 & 15.9 & & 510.0 & 30.8 \\
\hline 111.1 & 16.1 & & 511.0 & 20.9 \\
\hline 111.5 & 16.3 & & 512.0 & 36.2 \\
\hline 111.9 & 16.1 & 512.0 & 34.5 \\
\hline 112.0 & 16.6 & & 512.0 & 30.6 \\
\hline 112.0 & 16.0 & & 513.0 & 29.2 \\
\hline 112.0 & 16.3 & & 514.0 & 45.4 \\
\hline 112.7 & 16.3 & & 514.0 & 32.5 \\
\hline 113.1 & 15.4 & & 515.0 & 27.8 \\
\hline 116.0 & 15.5 & 516.0 & 50.7 \\
\hline 116.0 & 15.8 & & 516.0 & 36.4 \\
\hline 116.0 & 15.9 & 517.0 & 46.4 \\
\hline 116.3 & 15.3 & & 517.0 & 40.2 \\
\hline 116.5 & 15.5 & & 518.0 & 39.1 \\
\hline 116.5 & 15.5 & & 518.0 & 34.7 \\
\hline 119.6 & 15.5 & 519.0 & 30.6 \\
\hline 119.8 & 16.4 & & 521.0 & 38.5 \\
\hline 120.0 & 17.2 & 524.0 & 36.2 \\
\hline 120.5 & 18.7 & & 526.0 & 39.0 \\
\hline 120.7 & 17.8 & & 527.0 & 29.7 \\
\hline 122.8 & 19.5 & & 550.0 & 34.5 \\
\hline 122.8 & 19.2 & 553.0 & 31.6 \\
\hline 125.0 & 19.7 & 556.0 & 29.4 \\
\hline 126.7 & 20.0 & 557.0 & 37.0 \\
\hline 129.2 & 20.1 & & 558.0 & 30.8 \\
\hline & & & 560.0 & 38.2 \\
\hline & & & 560.0 & 34.8 \\
\hline & & 562.0 & 37.3 \\
\hline & & & 34.2 \\
\hline
\end{tabular}


Table 3. Phanerozoic seawater sulfate $\delta^{34} \mathrm{~S}$ curve

\begin{tabular}{|c|c|c|c|c|c|c|c|c|}
\hline Age & $\delta^{34} S_{s w^{a}}$ & $\delta^{34} S_{s w}{ }^{a}$ & $\delta^{34} S_{s w}{ }^{a}$ & {$\left[\mathrm{SO}_{4}{ }^{2-}\right]^{\mathrm{b}}$} & $\Delta^{34} S_{\text {sulf-py }}{ }^{c}$ & {$\left[\mathrm{SO}_{4}{ }^{2-}\right]^{\mathrm{d}}$} & {$\left[\mathrm{SO}_{4}{ }^{2-}\right]^{\mathrm{d}}$} & {$\left[\mathrm{SO}_{4}{ }^{2-}\right]^{\mathrm{d}}$} \\
\hline (Ma) & (\%o) & (\%o) & (\%o) & $\mathrm{mM}$ & $(\%)$ & $\mathrm{mM}$ & $\mathrm{mM}$ & $\mathrm{mM}$ \\
\hline & mean & -1 st.dev. & +1 st.dev. & $\max$ & & mean & -1 st.dev. & +1 st.dev. \\
\hline 0 & 21.2 & 20.9 & 21.5 & 364 & 45.8 & 20.5 & 11.8 & 35.7 \\
\hline 5 & 22.0 & 21.6 & 22.3 & 901 & 45.9 & 20.6 & 11.9 & 35.8 \\
\hline 10 & 22.0 & 21.7 & 22.3 & 2550 & 46.0 & 20.7 & 12.0 & 36.0 \\
\hline 15 & 22.0 & 21.7 & 22.3 & 1383 & 46.0 & 20.7 & 12.0 & 36.0 \\
\hline 20 & 21.8 & 21.4 & 22.2 & 1481 & 45.8 & 20.5 & 11.8 & 35.7 \\
\hline 25 & 21.7 & 21.3 & 22.1 & 942 & 45.5 & 20.2 & 11.6 & 35.2 \\
\hline 30 & 21.6 & 21.2 & 21.9 & 696 & 45.6 & 20.3 & 11.7 & 35.3 \\
\hline 35 & 22.0 & 21.6 & 22.5 & 817 & 45.8 & 20.5 & 11.8 & 35.7 \\
\hline 40 & 22.0 & 21.3 & 22.7 & 244 & 45.9 & 20.6 & 11.9 & 35.8 \\
\hline 45 & 21.6 & 20.7 & 22.4 & 121 & 44.4 & 19.0 & 10.8 & 33.4 \\
\hline 50 & 19.2 & 18.4 & 20.0 & 144 & 42.5 & 17.1 & 9.6 & 30.6 \\
\hline 55 & 18.1 & 17.2 & 18.9 & 297 & 41.8 & 16.5 & 9.1 & 29.6 \\
\hline 60 & 18.5 & 17.9 & 19.2 & 589 & 41.8 & 16.5 & 9.1 & 29.6 \\
\hline 65 & 18.9 & 18.5 & 19.3 & 1059 & 42.1 & 16.8 & 9.3 & 30.0 \\
\hline 70 & 18.9 & 18.5 & 19.3 & 802 & 42.3 & 17.0 & 9.5 & 30.3 \\
\hline 75 & 19.2 & 18.9 & 19.6 & 504 & 42.2 & 16.9 & 9.4 & 30.1 \\
\hline 80 & 18.9 & 18.4 & 19.4 & 478 & 41.4 & 16.1 & 8.9 & 29.0 \\
\hline 85 & 18.4 & 17.9 & 18.8 & 619 & 41.3 & 16.0 & 8.8 & 28.8 \\
\hline 90 & 18.5 & 17.9 & 19.1 & 274 & 39.5 & 14.4 & 7.8 & 26.3 \\
\hline 95 & 18.9 & 18.3 & 19.4 & 142 & 39.4 & 14.3 & 7.7 & 26.2 \\
\hline 100 & 17.3 & 16.3 & 18.2 & 173 & 40.1 & 14.9 & 8.1 & 27.1 \\
\hline 105 & 16.1 & 15.2 & 17.0 & 449 & 39.0 & 14.0 & 7.5 & 25.7 \\
\hline 110 & 16.2 & 15.6 & 16.7 & 400 & 38.6 & 13.6 & 7.3 & 25.1 \\
\hline 115 & 15.9 & 15.2 & 16.6 & 150 & 38.8 & 13.8 & 7.4 & 25.4 \\
\hline 120 & 17.1 & 16.0 & 18.1 & 101 & 39.6 & 14.5 & 7.8 & 26.5 \\
\hline 125 & 19.0 & 17.7 & 20.2 & 124 & 40.3 & 15.1 & 8.2 & 27.4 \\
\hline 130 & 17.2 & 15.4 & 19.0 & 172 & 40.5 & 15.3 & 8.4 & 27.7 \\
\hline 135 & 17.1 & 15.6 & 18.6 & 86 & 42.3 & 17.0 & 9.5 & 30.3 \\
\hline 140 & 18.1 & 14.9 & 21.3 & 60 & 43.4 & 18.0 & 10.2 & 31.9 \\
\hline 145 & 15.7 & 12.8 & 18.7 & 86 & 43.0 & 17.6 & 9.9 & 31.3 \\
\hline 150 & 16.7 & 14.3 & 19.1 & 236 & 42.6 & 17.2 & 9.7 & 30.7 \\
\hline 155 & 16.3 & 14.9 & 17.7 & 283 & 43.1 & 17.7 & 10.0 & 31.5 \\
\hline 160 & 16.5 & 15.3 & 17.8 & 185 & 43.8 & 18.4 & 10.4 & 32.5 \\
\hline 165 & 18.0 & 16.4 & 19.6 & 254 & 43.7 & 18.3 & 10.4 & 32.4 \\
\hline 170 & 18.5 & 17.2 & 19.8 & 442 & 43.9 & 18.5 & 10.5 & 32.7 \\
\hline 175 & 18.3 & 16.8 & 19.7 & 185 & 43.7 & 18.3 & 10.4 & 32.4 \\
\hline 180 & 18.9 & 16.5 & 21.2 & 87 & 45.0 & 19.7 & 11.3 & 34.4 \\
\hline 185 & 19.3 & 16.6 & 21.9 & 68 & 45.0 & 19.7 & 11.3 & 34.4 \\
\hline 190 & 18.4 & 15.7 & 21.1 & 83 & 45.2 & 19.9 & 11.4 & 34.7 \\
\hline 195 & 17.5 & 14.6 & 20.3 & 92 & 45.5 & 20.2 & 11.6 & 35.2 \\
\hline
\end{tabular}




\begin{tabular}{|c|c|c|c|c|c|c|c|c|}
\hline 200 & 17.9 & 15.0 & 20.9 & 75 & 45.6 & 20.3 & 11.7 & 35.3 \\
\hline 205 & 19.4 & 16.5 & 22.3 & 102 & 45.9 & 20.6 & 11.9 & 35.8 \\
\hline 210 & 19.3 & 16.9 & 21.7 & 221 & 46.2 & 20.9 & 12.1 & 36.3 \\
\hline 215 & 18.7 & 16.7 & 20.7 & 335 & 46.6 & 21.4 & 12.4 & 37.0 \\
\hline 220 & 18.7 & 16.7 & 20.7 & 477 & 47.3 & 22.1 & 12.9 & 38.1 \\
\hline 225 & 18.7 & 16.5 & 20.9 & 735 & 48.2 & 23.1 & 13.6 & 39.6 \\
\hline 230 & 18.8 & 16.0 & 21.5 & 284 & 48.1 & 23.0 & 13.6 & 39.5 \\
\hline 235 & 19.0 & 16.0 & 22.1 & 116 & 46.9 & 21.7 & 12.6 & 37.4 \\
\hline 240 & 19.8 & 16.3 & 23.3 & 96 & 46.4 & 21.1 & 12.3 & 36.6 \\
\hline 245 & 19.3 & 15.5 & 23.2 & 88 & 45.4 & 20.1 & 11.5 & 35.0 \\
\hline 250 & 17.7 & 13.3 & 22.0 & 75 & 44.8 & 19.4 & 11.1 & 34.1 \\
\hline 255 & 15.8 & 11.6 & 20.1 & 91 & 43.7 & 18.3 & 10.4 & 32.4 \\
\hline 260 & 15.4 & 11.5 & 19.3 & 105 & 41.0 & 15.7 & 8.7 & 28.4 \\
\hline 265 & 14.8 & 11.3 & 18.3 & 109 & 38.9 & 13.9 & 7.5 & 25.5 \\
\hline 270 & 15.4 & 11.7 & 19.0 & 197 & 38.4 & 13.5 & 7.2 & 24.9 \\
\hline 275 & 15.4 & 11.8 & 19.1 & 254 & 37.5 & 12.7 & 6.7 & 23.7 \\
\hline 280 & 15.0 & 11.6 & 18.4 & 165 & 35.8 & 11.4 & 5.9 & 21.6 \\
\hline 285 & 14.2 & 11.2 & 17.2 & 161 & 35.0 & 10.8 & 5.5 & 20.6 \\
\hline 290 & 13.6 & 11.0 & 16.1 & 210 & 34.5 & 10.4 & 5.3 & 20.0 \\
\hline 295 & 13.2 & 10.9 & 15.5 & 234 & 32.6 & 9.1 & 4.5 & 17.9 \\
\hline 300 & 13.5 & 11.3 & 15.6 & 239 & 33.0 & 9.4 & 4.6 & 18.3 \\
\hline 305 & 13.8 & 12.0 & 15.7 & 175 & 30.0 & 7.5 & 3.5 & 15.2 \\
\hline 310 & 14.4 & 12.6 & 16.1 & 126 & 30.2 & 7.6 & 3.6 & 15.4 \\
\hline 315 & 15.0 & 13.4 & 16.7 & 117 & 30.3 & 7.7 & 3.6 & 15.5 \\
\hline 320 & 15.3 & 13.7 & 16.9 & 135 & 30.2 & 7.6 & 3.6 & 15.4 \\
\hline 325 & 15.0 & 13.3 & 16.7 & 196 & 29.5 & 7.2 & 3.3 & 14.7 \\
\hline 330 & 14.6 & 13.0 & 16.3 & 403 & 29.0 & 6.9 & 3.2 & 14.2 \\
\hline 335 & 14.7 & 12.8 & 16.5 & 158 & 29.1 & 7.0 & 3.2 & 14.3 \\
\hline 340 & 15.1 & 12.8 & 17.4 & 74 & 29.1 & 7.0 & 3.2 & 14.3 \\
\hline 345 & 16.4 & 13.7 & 19.1 & 59 & 29.4 & 7.1 & 3.3 & 14.6 \\
\hline 350 & 17.8 & 15.0 & 20.6 & 50 & 27.5 & 6.1 & 2.7 & 12.8 \\
\hline 355 & 19.1 & 16.2 & 21.9 & 74 & 28.7 & 6.7 & 3.1 & 13.9 \\
\hline 360 & 19.4 & 16.6 & 22.2 & 196 & 29.7 & 7.3 & 3.4 & 14.9 \\
\hline 365 & 19.4 & 16.1 & 22.6 & 287 & 30.2 & 7.6 & 3.6 & 15.4 \\
\hline 370 & 19.7 & 16.1 & 23.4 & 158 & 30.3 & 7.7 & 3.6 & 15.5 \\
\hline 375 & 20.3 & 16.8 & 23.9 & 69 & 30.3 & 7.7 & 3.6 & 15.5 \\
\hline 380 & 20.5 & 17.3 & 23.7 & 38 & 30.4 & 7.7 & 3.6 & 15.6 \\
\hline 385 & 20.0 & 16.6 & 23.4 & 38 & 29.2 & 7.0 & 3.2 & 14.4 \\
\hline 390 & 21.3 & 17.7 & 24.9 & 74 & 29.0 & 6.9 & 3.2 & 14.2 \\
\hline 395 & 22.7 & 18.8 & 26.5 & 84 & 28.7 & 6.7 & 3.1 & 13.9 \\
\hline 400 & 24.1 & 20.4 & 27.7 & 67 & 29.4 & 7.1 & 3.3 & 14.6 \\
\hline 405 & 25.1 & 22.0 & 28.3 & 92 & 29.5 & 7.2 & 3.3 & 14.7 \\
\hline 410 & 25.3 & 22.2 & 28.4 & 177 & 29.6 & 7.2 & 3.4 & 14.8 \\
\hline 415 & 25.5 & 22.4 & 28.7 & 310 & 31.5 & 8.4 & 4.0 & 16.7 \\
\hline 420 & 25.6 & 22.6 & 28.6 & 276 & 32.0 & 8.7 & 4.2 & 17.3 \\
\hline 425 & 25.7 & 22.9 & 28.5 & 108 & 32.3 & 8.9 & 4.3 & 17.6 \\
\hline
\end{tabular}




\begin{tabular}{|r|r|r|r|r|r|r|r|r|}
\hline 430 & 26.4 & 23.1 & 29.7 & 45 & 32.8 & 9.3 & 4.5 & 18.1 \\
\hline 435 & 27.1 & 23.3 & 31.0 & 36 & 33.3 & 9.6 & 4.8 & 18.7 \\
\hline 440 & 25.9 & 21.8 & 30.0 & 54 & 31.9 & 8.7 & 4.2 & 17.1 \\
\hline 445 & 25.7 & 21.8 & 29.6 & 106 & 31.1 & 8.2 & 3.9 & 16.3 \\
\hline 450 & 26.2 & 22.3 & 30.1 & 104 & 30.0 & 7.5 & 3.5 & 15.2 \\
\hline 455 & 26.3 & 22.8 & 29.7 & 79 & 28.4 & 6.6 & 3.0 & 13.6 \\
\hline 460 & 25.6 & 21.9 & 29.3 & 93 & 27.9 & 6.3 & 2.8 & 13.2 \\
\hline 465 & 25.1 & 21.1 & 29.2 & 105 & 26.8 & 5.7 & 2.5 & 12.2 \\
\hline 470 & 25.6 & 21.7 & 29.5 & 161 & 29.0 & 6.9 & 3.2 & 14.2 \\
\hline 475 & 25.9 & 22.1 & 29.8 & 113 & 28.8 & 6.8 & 3.1 & 14.0 \\
\hline 480 & 26.7 & 22.5 & 30.9 & 63 & 29.4 & 7.1 & 3.3 & 14.6 \\
\hline 485 & 28.5 & 24.6 & 32.5 & 98 & 30.5 & 7.8 & 3.7 & 15.7 \\
\hline 490 & 29.6 & 25.2 & 33.9 & 207 & 31.1 & 8.2 & 3.9 & 16.3 \\
\hline 495 & 30.5 & 25.2 & 35.7 & 154 & 31.4 & 8.3 & 4.0 & 16.6 \\
\hline 500 & 31.8 & 25.8 & 37.8 & 113 & 31.2 & 8.2 & 3.9 & 16.4 \\
\hline 505 & 32.8 & 26.7 & 38.8 & 49 & 31.4 & 8.3 & 4.0 & 16.6 \\
\hline 510 & 33.5 & 27.4 & 39.5 & 37 & 31.0 & 8.1 & 3.9 & 16.2 \\
\hline 515 & 35.7 & 29.6 & 41.8 & 75 & 28.6 & 6.7 & 3.0 & 13.8 \\
\hline 520 & 36.3 & 30.8 & 41.8 & 199 & 28.9 & 6.8 & 3.1 & 14.1 \\
\hline 525 & 35.5 & 30.5 & 40.5 & 115 & 29.6 & 7.2 & 3.4 & 14.8 \\
\hline 530 & 34.7 & 29.6 & 39.8 & 186 & 29.9 & 7.4 & 3.5 & 15.1 \\
\hline 535 & 34.2 & 28.9 & 39.5 & 449 & 30.4 & 7.7 & 3.6 & 15.6 \\
\hline 540 & 33.7 & 28.7 & 38.8 & 281 & 22.1 & 3.6 & 1.4 & 8.5 \\
\hline 545 & 33.5 & 29.2 & 37.8 & 109 & 21.3 & 3.3 & 1.2 & 7.9 \\
\hline 550 & 33.4 & 30.0 & 36.7 & 38 & 18.9 & 2.5 & 0.8 & 6.3 \\
\hline 555 & 33.4 & 30.2 & 36.6 & 28 & 17.4 & 2.0 & 0.6 & 5.5 \\
\hline 560 & 34.4 & 31.4 & 37.4 & 39 & 16.7 & 1.9 & 0.5 & 5.1 \\
\hline 565 & 34.6 & 31.5 & 37.7 & 153 & 15.8 & 1.6 & 0.4 & 4.6 \\
\hline
\end{tabular}

Notes:

${ }^{a} \delta^{34} S_{s w}$ values for LOWESS curve calculated from data in Table 2; shown in Figure 3A.

${ }^{\mathrm{b}}\left[\mathrm{SO}_{4}{ }^{2-}\right]$ (max) calculated from $\delta^{34} \mathrm{~S}_{\mathrm{sw}}$ in col B using eqs 1-3; shown in Figure 4.

${ }^{c} \Delta^{34} S_{\text {sulf-py values }}$ from figure 3 of Wu et al. (2010), GCA 74:2053-2071; shown in Figure 3C.

${ }^{\mathrm{d}}\left[\mathrm{SO}_{4}{ }^{2-}\right]$ values calculated from MSR fractionation relationship (Eqs 5-7); shown in Figure 4. 
Table 4. Analysis of high-frequency seawater sulfate variation

\begin{tabular}{|c|c|c|c|c|c|c|c|c|}
\hline Rec. & Unit & Location & System & Age & $\mathbf{n}$ & $\Delta^{34} S_{\text {sulf-py }}$ & $\delta^{34} S_{\text {CAS }}$ shift & $\begin{array}{c}\partial \delta^{34} S_{\text {CAS }} / \partial \mathrm{t}( \\
\max )\end{array}$ \\
\hline & & & & (Ma) & & (\%o) & & (\%o $\left.\mathrm{Myr}^{-1}\right)$ \\
\hline & & & & & & & & \\
\hline & Neoproterozoic units (Fig 6) & & & & & & & \\
\hline $\mathrm{a}$ & Iwr Doushantuo Fm & China & up Proterozoic & $636-570$ & 40 & $11.6( \pm 6.3)$ & 38 to $24 \%$ at $636-633 \mathrm{Ma}$ & $5( \pm 2 X)$ \\
\hline $\mathrm{b}$ & Brachina Fm/Wilpena Grp & Namibia & up Proterozoic & $636-620$ & 2 & $31( \pm 5)$ & 18 to $42 \%$ at $636-620 \mathrm{Ma}$ & $1.5( \pm 2 X)$ \\
\hline $\mathrm{c}$ & Maieberg Fm/Otavi Grp & Namibia & up Proterozoic & $636-620$ & 5 & $11.2( \pm 15.2)$ & 31 to $14 \%$ at $\sim 636-635 \mathrm{Ma}$ & $17( \pm 3 X)$ \\
\hline $\mathrm{d}$ & Sonora succession & Sonora & Iwr Ediacaran & $600-580$ & 11 & $7.4( \pm 4.2)$ & 17 to $29 \%$ over 2 Myr & $6( \pm 3)$ \\
\hline $\mathrm{e}$ & Wonoka Fm/Wilpena Grp & Namibia & up Proterozoic & $585-581$ & 4 & $39( \pm 6)$ & 22 to $18 \%$ ot $585-581 \mathrm{Ma}$ & $1.0( \pm 3 X)$ \\
\hline$f$ & Sonora succession & Sonora & up Ediacaran & $580-542$ & 8 & $14.6( \pm 7.0)$ & 33 to $18 \%$ over $\sim 4 \mathrm{Myr}$ & $4( \pm 2)$ \\
\hline $\mathrm{g}$ & up Doushantuo Fm & China & up Proterozoic & $570-551$ & 17 & $23.9( \pm 9.3)$ & 35 to $11 \%$ at $568-551$ & $1.3( \pm 2 X)$ \\
\hline $\mathrm{h}$ & Zarls Fm/Nama Grp & Namibia & up Proterozoic & $555-542$ & 18 & $5.8( \pm 3.4)$ & 30 to $70 \%$ at $549-548 \mathrm{Ma}$ & $40( \pm 2 x)$ \\
\hline $\mathrm{j}$ & upper Huqf Supergroup & Oman & up Proterozoic-Cambrian & $>580-540$ & 70 & $30.0( \pm 5.7)$ & 23 to $43 \%$ at $548-547 \mathrm{Ma}$ & $20( \pm 2 X)$ \\
\hline \multirow[t]{2}{*}{$\mathrm{k}$} & Death Valley succession & Death Valley & Proterozoic-Cambrian & $544-542$ & 30 & $11.3( \pm 7.1)$ & 24 to $37 \%$ over $\sim 1.2 \mathrm{Myr}$ & $11( \pm 6)$ \\
\hline & Paleozoic units (Fig 7) & & & & & & & \\
\hline$m$ & Sonora succession & Sonora & Cambrian/Terreneuvian & $542-520$ & 6 & $15.7( \pm 6.5)$ & 34 to $8 \%$ in $\sim 4 \mathrm{Myr}$ & $7( \pm 4)$ \\
\hline $\mathrm{n}$ & Sonora succession & Sonora & Cambrian/Series 2-3 & $520-505$ & 3 & $11.3( \pm 8.5)$ & 8 to $38 \%$ in $~ 1.4 \mathrm{Myr}$ & $22( \pm 11)$ \\
\hline $\mathrm{n}^{\prime}$ & Death Valley succession & Death Valley & Cambrian/Series 2-3 & $520-505$ & 1 & 11 & 31 to $14 \%$ in $\sim 0.8 \mathrm{Myr}$ & $23( \pm 11)$ \\
\hline$p$ & Lancara Fm-Genestosa & Spain & Lower-Middle Cambrian & $520-505$ & 19 & $16.1( \pm 3.6)$ & 18 to $27 \%$ o in 1 Myr & $9( \pm 3 X)$ \\
\hline$p^{\prime}$ & Lancara Fm-Cremenes & France & Lower-Middle Cambrian & $520-505$ & 36 & $10.8( \pm 4.4)$ & 22 to $29 \%$ in $\sim 0.3 \mathrm{Myr}$ & $20( \pm 3 X)$ \\
\hline$q$ & Spice excursion & Australia & Upper Cambrian & $499-494$ & 11 & $26.0( \pm 9.8)$ & 63 to $35 \%$ at $495.4-494.0 \mathrm{Ma}$ & $20( \pm 1.5 X)^{b}$ \\
\hline $\mathrm{q}^{\prime}$ & Spice excursion & Missouri, USA & Upper Cambrian & $499-494$ & 8 & $28.8( \pm 10.1)$ & 38 to $27 \%$ at $496.2-495.2 \mathrm{Ma}$ & $11( \pm 1.5 X)^{b}$ \\
\hline \multirow[t]{2}{*}{ q" } & Spice excursion & Nevada, USA & Upper Cambrian & $499-494$ & 2 & $2.1( \pm 4.9)$ & 46 to $33 \%$ at $494.9-493.3 \mathrm{Ma}$ & $8( \pm 1.5 X)^{b}$ \\
\hline & Meso-Cenozoic units (Fig 8) & & & & & & & \\
\hline$r$ & Nanpanjiang Basin carbs & China & Lower Triassic & $252-250$ & 143 & $36( \pm 4)$ & 25 to $33 \%$ over $\sim 0.3 \mathrm{Myr}$ & $25( \pm 2 X)$ \\
\hline $\mathrm{s}$ & Bravaisberget $\mathrm{Fm}$ & Spitsbergen & Middle Triassic & $245-238$ & 40 & $15.1( \pm 5.6)$ & 14 to $22 \%$ over $1.2 \mathrm{Myr}^{\mathrm{d}}$ & $4( \pm 2 X)$ \\
\hline $\mathrm{t}$ & Toarcian succession & England & Lower Jurassic & $183-178^{c}$ & $\sim 60$ & $51( \pm 6)^{e}$ & 16.5 to $22.5 \%$ o in $300 \mathrm{kyr}$ & $20( \pm 4)$ \\
\hline $\mathrm{t}$ & Toarcian succession & England & Lower Jurassic & $183-178^{c}$ & 55 & $51( \pm 6)^{\mathrm{e}}$ & 16 to $22 \%$ in $300 \mathrm{kyr}$ & $20( \pm 4)$ \\
\hline $\mathrm{t}^{\prime}$ & Toarcian succession & Tibet & Lower Jurassic & $183-178^{c}$ & 25 & $51( \pm 6)^{e}$ & 19 to $37 \%$ in $300 \mathrm{kyr}(?)$ & $60( \pm 30)(?)$ \\
\hline $\mathrm{v}$ & Early Cretaceous & South Atlantic & Lower Cretaceous & $120-118$ & $\sim 100$ & $46( \pm 4)^{f}$ & 15.5 to $20.0 \%$ in $1.2 \mathrm{Myr}$ & $12-16$ \\
\hline$w$ & Cenomanian-Turonian & Colorado, USA & Middle Cretaceous & $94-93$ & 22 & $46( \pm 4)^{f}$ & 12 to $19 \%$ o in $400 \mathrm{kyr}$ & $15-20$ \\
\hline$w^{\prime}$ & Cenomanian-Turonian & England & Middle Cretaceous & $94-93$ & $\sim 50$ & $45( \pm 7)^{f}$ & 18 to $22 \%$ in $600 \mathrm{kyr}$ & $6-7$ \\
\hline$w^{\prime \prime}$ & Cenomanian-Turonian & Italy & Middle Cretaceous & $94-93$ & $\sim 80$ & $47( \pm 7)^{f}$ & 20 to $24 \%$ in $600 \mathrm{kyr}$ & $6-7$ \\
\hline$z$ & Sapropel & Mediterranean & Pleistocene & $1.8-1.4$ & 11 & $+60.4( \pm 4.8)$ & $\mathrm{N} / \mathrm{A}$ & $<0.5^{g}$ \\
\hline
\end{tabular}


Table 4. Analysis of high-frequency seawater sulfate variation (cont.)

\begin{tabular}{|c|c|c|c|c|}
\hline Rec. & Ocean model a & $\begin{array}{c}{\left[\mathrm{SO}_{4}{ }^{2-}\right]_{\mathrm{sw}}} \\
\text { (Rate method) }\end{array}$ & $\begin{array}{c}{\left[\mathrm{SO}_{4}{ }^{2-}\right]_{\mathrm{sw}}} \\
\text { (MSR-trend method) }\end{array}$ & Source \\
\hline & & $(\mathrm{mM})$ & $(\mathrm{mM})$ & \\
\hline \multicolumn{5}{|c|}{ Neoproterozoic units (Fig 6) } \\
\hline$a$ & 0 & $0.5-6$ & $0.1-3$ & McFadden et al. (2008) \\
\hline $\mathrm{b}$ & 0 & $8-52$ & $5-16$ & Hurtgen et al. (2005) \\
\hline c & 0 & $<0.1-3$ & $<0.1-7$ & Hurtgen et al. (2006) \\
\hline$d$ & $A$ & $0.2-1.2$ & $<0.1-1.0$ & Loyd et al. (2012) \\
\hline $\mathrm{e}$ & A & $25->100$ & $24-70$ & Hurtgen et al. (2005) \\
\hline $\mathrm{f}$ & $\mathrm{A}$ & $3-22$ & $0.5-11$ & Loyd et al. (2012) \\
\hline $\mathrm{g}$ & $\mathrm{A}$ & $13-100$ & 3-35 & McFadden et al. (2008) \\
\hline $\mathrm{h}$ & A & $0.1-1.0$ & $<0.1-1.6$ & Ries et al. (2009) \\
\hline j & A & $1.5-8$ & $12-45$ & Fike \& Grotzinger (2008) \\
\hline $\mathrm{k}$ & $\mathrm{A}$ & $0.3-4$ & $0.1-8$ & Loyd et al. (2012) \\
\hline \multicolumn{5}{|c|}{ Paleozoic units (Fig 7) } \\
\hline $\mathrm{m}$ & A & $2-18$ & $0.8-14$ & Loyd et al. (2012) \\
\hline $\mathrm{n}$ & $\mathrm{A}$ & $0.2-4$ & $<0.1-9$ & Loyd et al. (2012) \\
\hline $\mathrm{n}^{\prime}$ & $\mathrm{A}$ & $0.7-3.5$ & $1.2-2.8$ & Loyd et al. (2012) \\
\hline$p$ & $\mathrm{~A}$ & $1.2-14$ & $2-8$ & Wotte et al. (2012) \\
\hline$p^{\prime}$ & $A$ & $0.3-5$ & $0.3-6$ & Wotte et al. (2012) \\
\hline$q$ & A & $1.2-6$ & $4-40$ & Gill et al. (2011a) \\
\hline$q^{\prime}$ & A & $2-12$ & $5-13$ & Gill et al. (2011a) \\
\hline$q^{\prime \prime}$ & A & $0.1-2.5$ & $2-6$ & Gill et al. (2011a) \\
\hline \multicolumn{5}{|c|}{ Meso-Cenozoic units (Fig 8) } \\
\hline$r$ & $\mathrm{O}$ & $0.5-2.5$ & $8-20$ & Song et al. (2014) \\
\hline $\mathrm{s}$ & $\mathrm{A}$ & $3-22$ & $1-10$ & Karcz (2010) \\
\hline $\mathrm{t}$ & $\mathrm{O}$ & $1.5-3$ & $18-50$ & Gill et al. (2011b) \\
\hline $\mathrm{t}$ & $\mathrm{O}$ & $1.5-3$ & $18-50$ & Newton et al. (2011) \\
\hline $\mathrm{t}^{\prime}$ & $\mathrm{O}$ & $0.5-2$ & $18-50$ & Newton et al. (2011) \\
\hline $\mathrm{v}$ & 0 & $2.5-4$ & $15-30$ & Wortmann \& Chernyavsky (2007) \\
\hline w & $\mathrm{O}$ & $1.5-3$ & $15-30$ & Adams et al. (2010) \\
\hline$w^{\prime}$ & 0 & $5-8$ & $12-40$ & Owens et al. (2013) \\
\hline w" & 0 & $5-8$ & $12-40$ & Owens et al. (2013) \\
\hline $\mathrm{z}$ & 0 & $60-120$ & $\mathrm{n} / \mathrm{a}$ & Scheiderich et al. (2010) \\
\hline
\end{tabular}




\section{Notes:}

${ }^{a}$ Compared to the oxic $(\mathrm{O})$ ocean model, the anoxic $(\mathrm{A})$ model yields seawater sulfate concentrations that are larger by a factor of $2.4 \mathrm{X}$.

b Rates also given in Table 5 of Gill et al. (2007)

${ }^{c}$ Age control from McArthur et al. (2000).

${ }^{d} \partial \delta^{34} S_{\text {CAS }} / \partial t(\max )$ estimated from Song et al. (2014).

e CAS $\delta^{34}$ S values are $16.4 \%$ and $37 \%$ for the tenuicostatum and falciferum zones, respectively. Pyrite $\delta^{34}$ S values are $-37 \pm 5 \%$ and $-12 \pm 3 \%$ o for the same zones, respectively (from Berner et al., 2013).

${ }^{\mathrm{f}}$ Based on Cretaceous pyrite $\delta^{34} \mathrm{~S}$ data of Strauss $(1997,1999)$

g Based on Cenozoic sulfate $\delta^{34} \mathrm{~S}$ data of Paytan et al. (1998). 